\title{
Identification of Potential Biomarkers For The Prognosis, Diagnosis, And Targeted Therapy in Kidney Renal Clear Cell Carcinoma
}

\section{Fang Cheng}

Union Hospital, Huazhong University of Science and Technology

\section{Qiang Li}

Union Hospital, Huazhong University of Science and Technology

Jinglin Wang

Union Hospital, Huazhong University of Science and Technology

Yumei Wang

Union Hospital, Huazhong University of Science and Technology

\section{Zhendi Wang}

Union Hospital, Huazhong University of Science and Technology

\section{Fang Zeng}

Huazhong University of Science and Technology

\section{Yu Zhang ( $\nabla$ zhangwkp@163.com)}

Union Hospital, Huazhong University of Science and Technology

\section{Research Article}

Keywords: kidney renal clear cell carcinoma, GEO; biomarker, TCGA, prognosis

Posted Date: November 23rd, 2021

DOl: https://doi.org/10.21203/rs.3.rs-1005845/v2

License: (c) (1) This work is licensed under a Creative Commons Attribution 4.0 International License. Read Full License 


\section{Abstract}

Background: Kidney renal clear cell carcinoma (KIRC) is the most common renal cell carcinoma types. This work aims to find potential diagnostic biomarkers and explore the biological functions related to the prognosis of KIRC.

Method: First, Gene expression profiles of GSE15641, GSE72304, GSE71963, GSE53757, and GSE36895 from GEO database. Differentially expressed genes (DEGs) were identified by the limma package in $R$ software. Next, gene ontology (GO) and Kyoto Encyclopedia of Gene and Genome (KEGG) pathway analysis were performed. Then protein-protein interaction (PPI) and hub genes were visualized by Cytoscape with STRING database. Then, we evaluate the predictive potential of hub genes expressions in KIRC with TCGA data. In addition, the relevant biological functions were identified using GSEA. Finally, we examined the differences of hub genes expression between multiple tumor tissues and normal tissues.

Results: A total of 141 DEGs (including 99 upregulated and 42 downregulated genes) were identified. GO analysis indicated that DEGs were mainly involved in oxidation-reduction process and response to hypoxia. The KEGG analysis primarily related to PPAR signaling pathway, and HIF-1 signaling pathway. Moreover, the PPI analysis revealed 5 hub genes (AOX1, ALDH6A1, ABAT, HADH, and PCCA). The 5 hub genes were significantly correlated with KIRC progression and might have prognostic significance for KIPC patients. And low expression of the hub genes associated biological pathways were enriched in the NF-KB activation, focal adhesion, and JAK-STAT signaling pathway, respectively.

Conclusion: Our study demonstrated that AOX1, ALDH6A1, ABAT, HADH, and PCCA can be used as prognostic biomarkers for KIRC.

\section{Introduction}

Kidney cancer remains one of the malignant tumors with the incidence increased notably in recent years(1). Renal cell carcinoma (RCC) is a malignant tumor originated from the renal epithelium, which can be further divided into several pathological subtypes(2). Among them, kidney clear cell renal cell carcinoma (KIRC) is the most common subtype, accounting for about $75 \%$ of all cases (3). Despite significant advances in modalities of diagnosis and treatment in the recent decades, however, at the first diagnosis, about $20-30 \%$ patients already have locally advanced or metastatic disease(4). Nevertheless, the median survival of patients with metastatic KIRC is about 13 months, and the 5 -year survival rate is less than $10 \%(5)$, which mainly metastatic patients that benefit few from currently available therapies. $\mathrm{KIRC}$ is a complicated disease, and combining the biomarkers with conventional clinical pathological predictors may have important clinical significance. Therefore, it is urgently necessary to find promising diagnostic biomarkers for KIRC.

In recent years, bioinformatics analysis based on microarray and high-throughput sequencing technology has been widely used to identify differentially expressed genes (DEGs) and functional pathways related to the occurrence and development of cancer(6). Previous studies identified CCND1 and PECAM1/CD31, 
which were closely correlated with the diagnosis and prognosis of KIRC(7). Lin et al identified CCL5 was a potential biomarker and therapeutic target which related to CD8+ T cell infiltration in KIRC (8). However, it is difficult to get reliable results from independent microarrays or high-throughput sequencing due to high false-positive rates. With the development of analytical technology, integrated bioinformatics analyses have been developed to analyze cross-platform microarray or high-throughput data on a large scale.

In this study, we downloaded five gene expression profiles (GSE15641, GSE72304, GSE71963, GSE53757, and GSE36895) from the GEO database. DEGs were identified by the limma package of R software. Then, the DAVID website was applied to GO analysis and KEGG pathway. Then PPI and hub genes were visualized by Cytoscape with STRING website. In addition, we examined the differences of hub genes expression between multiple tumor tissues and normal tissues via exploring TIMER, and Oncomine databases. Then, we evaluate the predictive potential of hub genes expressions in KIRC. Finally, the relevant biological functions were identified with GSEA. In conclusion, this study provides a new direction for the prognosis and treatment of patients with KIRC.

\section{Results}

\section{Identification of DEGs}

Firstly, all the genes from GSE15641, GSE72304, GSE71963, GSE53757, and GSE36895 were shown in the volcano plots (Figure 1A). In this study, $173 \mathrm{KIRC}$ tissues and 138 normal kidney tissues from five GEO data sets were involved. A total of 1760 upregulated and 421 downregulated genes were filtered from GSE15641; 962 upregulated and 396 downregulated genes from GSE72304; 1580 upregulated and 1484 downregulated genes from GSE71963; 1593 upregulated and 1985 downregulated genes from GSE53757; 910 upregulated and 1082 downregulated genes from GSE36895 based on the threshold of $\mathrm{P}<0.05$ and $|\log \mathrm{FC}|>1$, respectively. Subsequently, 99 common upregulated genes (Figure 1B) and 42 common downregulated genes (Figure 1C) were selected among the DEGs with Venn software online. The top 25 upregulated and downregulated genes were shown in Heat map with R package “ComplexHeatmap" (Figure 2).

\section{GO and KEGG pathway analyses}

GO analysis of the 141 DEGs (99 upregulated genes and 42 downregulated genes) was performed with the DAVID online analysis tool. In biological process (Figure 3A), the DEGs were significantly enriched in oxidation-reduction process (G0:0055114), response to hypoxia (G0:0001666), gluconeogenesis (G0:0006094), cellular response to hypoxia (G0:0071456), response to drug (G0:0042493), and lung development (GO:0030324). In cellular component terms (Figure 3B), the DEGs were mainly involved in the extracellular exosome (G0:0070062), extracellular space (G0:0005615), extracellular region (GO:0005576), protein complex (G0:0043234), and extracellular matrix (G0:0031012). For molecular functions (Figure 3C), the DEGs were mainly enriched in iron ion binding (G0:0005506), transporter activity (GO:0005215), fatty acid binding (GO:0005504), L-ascorbic acid binding (GO:00314180), and receptor binding (G0:0005102). The KEGG pathway analysis results were shown in Figure 3D, and it 
demonstrated that these targets were particularly enriched in PPAR signaling pathway, carbon signaling pathway, HIF-1 signaling pathway, valine, leucine ang isoleucine degradation, and gluconeogenesis.

\section{PPI network and hub gene selection}

The PPI network was constructed for further investigation of the interaction among the DEGs used Cytoscape software, based on the STRING database. The PPI network consisted of 104 nodes and 220 edges. 5 central nodes modules of the PPI networks were selected with Cytotype MCODE (Figure 4). The identified hub genes were AOX1, ALDH6A1, ABAT, HADH, and PCCA.

\section{Hub gene validation}

To further evaluate the role of the five candidate hub genes in KIRC prediction, TCGA_KIRC database was selected for validation. The results showed that the expression of these five hub genes were significantly downregulated in KIRC, which consistent with the mRNA level from GEO dataset (Figure 5A). To further clarify the clinical significance of AOX1, ALDH6A1, ABAT, HADH, and PCCA expression in KIRC, we analyzed their expression in different stages of KIRC. The low expression of hub genes was closely related with advanced tumor histology stage (Figure 5B), pathological stage (Figure 5C), distant metastasis (Figure 6A), and lymph node metastasis (Figure 6B), tumor status (Figure 6C) in KIRC. These results demonstrated that the low expression of AOX1, ALDH6A1, ABAT, HADH, and PCCA might be associated with KIRC progression and have prognostic significance for KIRC patients.

\section{Prognostic significance of hub genes in patients with KIRC}

We investigated the R package "survival" for the prognostic significance (overall survival and disease specific survival) of AOX1, ALDH6A1, ABAT, HADH, and PCCA expression in KIRC. These findings indicated that low expression of AOX1, ALDH6A1, ABAT, HADH, and PCCA predicted poor prognostic in in patients with KIRC (Figures 7A and 7B), which suggested that AOX1, ALDH6A1, ABAT, HADH, and PCCA were significant factors for predicting worse prognostic in KIRC.

\section{ROC curve analysis of hub genes}

ROC curve analysis was performed on five hub genes with the R package of " $p R O C$ ". AUC $>0.90$ was selected as the cutoff value, and it was found that three of the five hub genes with AUC $>90 \%$ included ALDH6A1 (AUC=0.939), HADH (AUC=0.979), and PCCA (AUC=0.931), respectively. The expression of ALDH6A1, HADH and PCCA have high accuracy in distinguishing normal kidney tissue from KIRC tissue, which has important significance for the accurate diagnosis of KIRC (Figure 8).

\section{GSEA analysis}

To further identify the possible mechanism of the hub genes in KIRC, GSEA was conducted to obtain the biological pathway. The TCGA_KIRC data set was divided into high and low-expression groups based on the median value of AOX1, ALDH6A1, ABAT, HADH, and PCCA, respectively. As shown in Figure 9, the 
result revealed that the low expression of AOX1 was enriched in the "REACTOME_FCERI_MEDIATED_NFKB activation", ALDH6A1 was enriched in the "KEGG_CYTOKINE_CYTOKINE_RECEPTOR_INTERACTION", ABAT was enriched in the "KEGG_FOCAL_ADHESION", HADH was enriched in the "REACTOME_SIGNALING_B_CELL_RECEPTOR", and PCCA was enriched in the "KEGG_JAKSTAT_SIGNALING_PATHWAY", respectively.

\section{The hub genes expression in different cancer types}

We applied the Oncomine database to analyze the expression of AOX1, ALDH6A1, ABAT, HADH, and PCCA. The results showed that these genes expression was significantly different in various cancer types (Figure 10A). And AOX1, ALDH6A1, ABAT, HADH, and PCCA expression were significantly low in KIRC, compared with the corresponding normal tissues (Figure 10B).

\section{Discussion}

Because of the aggressiveness and recurrence of KIRC, treatment of KIRC remains a great challenge. KIRC has no symptoms in the early stage, which is difficult to screen, and it needs to be effectively managed after diagnosis. Therefore, it is necessary to explore the pathogenesis and prognosis biomarkers for KIRC. In this study, we performed a comprehensive bioinformatics analysis to identify the potential molecular mechanism and explore the potential prognostic value of biomarkers of KIRC. First, we obtained the gene expression from five GEO database (GSE15641, GSE72304, GSE71963, GSE53757, and GSE36895). As a result, 99 upregulated and 42 downregulated genes were regarded as DEGs.

Hypoxia/oxygen sensing signal plays a key role in the regulation of cancer progression $(9,10)$. Previous studies have shown that HIF pathway plays these functions in cancer by affecting cell proliferation, cell death, angiogenesis, cell adhesion molecules and immunosuppressive factors $(11,12)$. In addition, most cancer cells exhibit abnormal activation of lipid metabolism, which induces tumor synthesis and promotes tumor occurrence, proliferation and progression(13). The functional enrichment analysis indicated that the DEGs were mainly enriched in oxidation-reduction process, response to hypoxia, gluconeogenesis, extracellular exosome, fatty acid binding, and so on. The KEGG pathway analysis results indicated that the DEGs were particularly enriched in PPAR signaling pathway and HIF-1 signaling pathway.

Furthermore, 5 hub genes among the common DEGs were identified by constructing a PPI network, containing AOX1, ALDH6A1, ABAT, HADH, and PCCA. Subsequently, the hub genes were validated in TCGA data set, which the expression of hub genes tendency were consistent with the GEO data set. Compared to normal tissues, we found that AOX1, ALDH6A1, ABAT, HADH, and PCCA expression was highly down-regulated in KIRC tissues. In addition, AOX1, ALDH6A1, ABAT, HADH, and PCCA expression were closely associated with advanced pathological stage, tumor histology stage, tumor status, distant metastasis, and lymph node metastasis in KIRC. These results indicated that AOX1, ALDH6A1, ABAT, $\mathrm{HADH}$, and PCCA played a significant role in the progression and metastasis of KIRC. Furthermore, low AOX1, ALDH6A1, ABAT, HADH, and PCCA expression was associated with poor prognosis in KIRC. Our 
results strongly suggested that AOX1, ALDH6A1, ABAT, HADH, and PCCA could serve as a promising prognostic biomarker for KIRC.

AOX1 is an oxidase with a wide range of substrates, which is involved in the biological activity of prodrugs, the regulation of oxygen species homeostasis, the production of nitric oxide and lipogenesis(14). The expression level of AOX1 gene is associated with the occurrence of a variety of cancers. The loss of AOX1 in advanced bladder cancer promotes the progression of cancer(15). Similarly, AOX1 transcriptionally activates CD133 through inositol phosphate 3-kinase (PI3K)/Akt pathway and participates in the occurrence of colorectal cancer(16). Recent study showed that AOX1 was identified as a prognostic factor for KIRC patients(17). Aminobutyric acid transaminase (ABAT) and Aldehyde dehydrogenase 6 family, member A1 (ALDH6A1) are involved in the pyruvate metabolism and catabolism of valine, leucine, and isoleucine(18, 19). ALDH6A1 expression was found to be markedly downregulated in KIRC tissues, in association with poor survival(20). Accordingly, its overexpression significantly decreased cell proliferation and migration and impaired oncologic metabolism in KIRC cells(21). Previous study has indicated that low-expressed ABAT and ALDH6A1 might be promising diagnostic and prognostic biomarkers for $\mathrm{KIRC}(22)$.

Mitochondrial short chain L-3-Hydroxyacyl-CoA dehydrogenase (HADH) is a gene coding for an enzyme involved in the penultimate step of the $\beta$-oxidation pathway(23). Clinical studies proved that the mRNA levels of several enzymes involved in $\beta$-oxidation were decreased in cancer. Previous study has shown that downregulation of HADH promoted gastric cancer progression via activation of Akt signaling pathway(24). Propionyl-CoA carboxylase (PCC) is a mitochondrial, biotin-dependent enzyme that functions in the catabolism of branched-chain amino acids, fatty acids with odd-numbered chain lengths, and other metabolites(25). PCCA signature was a promising prognostic marker with a good prospect of clinical application for HER-2 negative breast cancer patients(26). This is the first time to clarify important prognostic role of HADH and PCCA in KIRC.

Furthermore, GSEA analysis revealed that the low expression of AOX1 was enriched in the "REACTOME_FCERI_MEDIATED_NF-KB activation", ALDH6A1 was enriched in the "KEGG_CYTOKINE_CYTOKINE_RECEPTOR_INTERACTION", ABAT was and enriched in the "KEGG_FOCAL_ADHESION", HADH was and enriched in the "REACTOME_SIGNALING_B_CELL_RECEPTOR", and PCCA was and enriched in the "KEGG_JAKSTAT_SIGNALING_PATHWAY", respectively.

NF-kB pathway involved in many processes that play a key role in the development and progression of cancer such as proliferation, migration and apoptosis $(27,28)$. Aberrant or constitutive NF-KB activation has been detected in many human malignancies. In recent years, NF-kB has turned out to be an interesting therapeutic target for cancer treatment(29). Focal adhesion signaling hubs are composed of a large variety of pro-survival signaling molecules including integrins, growth factor receptors and intracellular molecules that critically regulate cell behavior, impact on tumor cell survival and serve as potential cancer targets $(30,31)$. The JAK/ STAT pathway played a crucial role in many biological 
functions during the multistep development of human tumors, including proliferation, inflammation, and survival $(32,33)$.

\section{Conclusion}

In conclusion, five-mRNA signature (AOX1, ALDH6A1, ABAT, HADH, and PCCA) predicting the prognosis of KIRC. The study may have some limitations. In fact, this study is only a preliminary part at present, which need further verification with independent datasets and carry out experiments in the future. In addition, we have not clarified the mechanism through which AOX1, ALDH6A1, ABAT, HADH, and PCCA suppresses KIRC progression in this study. Our future research may focus on the molecular mechanism for AOX1, ALDH6A1, ABAT, HADH, and PCCA dysregulation in KIRC.

\section{Materials And Methods}

\section{Acquisition of data of gene expression profiles}

GEO (https://www.ncbi.nlm.nih.gov/geo/) database is regarded as a free public database of microarray gene profile (34) and we obtained the gene expression profile of GSE15641, GSE72304, GSE71963, GSE53757, and GSE36895 in KIRC and normal kidney tissues. GSE15641 was based on the GPL96 platform ([HG-U133A] Affymetrix Human Genome U133A Array), containing 23 normal kidney tissues and 32 KIRC specimens. GSE72304 was based on the GPL10332 platform (Agilent-026652 Whole Human Genome Microarray), consisting of 4 normal endometrium tissues and 8 KIRC tissues. The platform of GSE71963 was the GPL6480 (Agilent-014850 Whole Human Genome Microarray), which included 16 normal controls and 32 KIRC samples. The GSE53757 and GSE36895 were based on the GPL570 platform (GPL570 [HG-U133_Plus_2] Affymetrix Human Genome U133 Plus 2.0 Array), included 72 KIRC tissues and 72 normal kidney tissues; and $29 \mathrm{KIRC}$ tissues and 23 normal kidney tissues, respectively. The data set information was shown in Table 1. The downloaded data were processed with the $\mathrm{R}$ package, then calibrated, standardized, and log2 transformation on all data of gene expression. 
Table 1

Details of the four Gene Expression Omnibus KIRC data sets.

\begin{tabular}{|llllc|}
\hline GEO & Tissue & Platform & Normal & Tumor \\
\hline GSE15641 & Kidney & $\begin{array}{l}\text { GPL96 [HG-U133A] Affymetrix Human Genome U133A } \\
\text { Array }\end{array}$ & 23 & 32 \\
GSE72304 & Kidney & $\begin{array}{l}\text { GPL10332 Agilent-026652 Whole Human Genome } \\
\text { Microarray }\end{array}$ & 4 & 8 \\
\hline GSE71963 & Kidney & $\begin{array}{l}\text { GPL6480 Agilent-014850 Whole Human Genome } \\
\text { Microarray }\end{array}$ & 16 & 32 \\
\hline GSE36895 & Kidney & $\begin{array}{l}\text { GPL570 [HG-U133_Plus_2] Affymetrix Human Genome } \\
\text { G133 Plus 2.0 Array }\end{array}$ & 72 & 72 \\
\hline
\end{tabular}

\section{Screening of DEGs}

DEGs between KIRC samples and normal kidney samples were identified with the R package "DEseq2". The $|\log \mathrm{FC}|>1$ and adjust P.value $<0.05$ were selected as the threshold for each group. Gene differential analysis from the five microarray was conducted with Volcano plots. Then, the extracted DEGs were checked in Venn software online (http://bioinformatics.psb.ugent.be/webtools/Venn/) to identify the common DEGs among the five datasets. The DEGs with logFC $>0$ was considered as up-regulated genes, while $\log \mathrm{FC}<0$ was down-regulated genes.

\section{GO and KEGG analysis}

To investigate the underlying mechanisms and biological functions of potential prognosis gene in KIRC, the $\mathrm{GO}$ and biological pathways enriched with differentially expressed genes were annotated, classified, and analysed for enrichment using the Diversity Visualization Integrated Database (DAVID 6.8; https://david.ncifcrf.gov/) (35). A KEGG pathway enrichment analysis was performed based on the KOBAS (kobas.cbi.pku.edu.cn/) database (36). P.value $<0.05$ and counts more than 2 were considered as statistically significant difference and significant enrichment, respectively.

\section{PPI network analysis}

To further visualize and evaluate interactions among DEGs, the STRING online database (https://stringdb.org/cgi/input.pl) (37) was applied to examine the potential correlation between these DEGs with the nodes association confidence score $>0.4$. Then, the network analysis software Cytoscape (www.cytoscape.org; version 3.7.2) was used to visualize networks (38). And the plugin Molecular Complex Detection (MCODE) in Cytoscape was used to identify the hub gene in functional networks. 


\section{Validation of the hub genes in TCGA database}

The expression of the hub genes was further validated in the TCGA_KIRC data set. KIRC patient datasets, with gene expression profiles and paired clinical information, were downloaded from the publicly available TCGA, and included 539 tumor tissues and 72 para-cancerous tissue. The R package "ggplot2" was applied to evaluate the correlation between hub genes expression and clinicopathologic information (different pathological stages) in KIRC. And the R package "survival" was used to evaluate the prognostic values of hub genes in KIRC.

\section{ROC curve of hub genes}

The receiver operating characteristic $(\mathrm{ROC})$ curve was used to evaluate the predictive power of the signature with R package " $p R O C$ ". AUC $>90 \%$ was set as the cutoff value to determine the diagnostic significance of Hub Genes.

\section{GESA}

We analyzed the association among hub genes expression, biological processes/pathways and phenotypes with GSEA (https://www.gsea-msigdb.org/gsea/index.jsp)(39). Gene sets with FDR $<0.05$ were considered significantly enriched.

\section{Oncomine database analysis}

Oncomine database (www.oncomine.org) a publicly accessible online database providing powerful, genome-wide expression analysis with cancer microarray information(40). The Oncomine database was used to analyze the hub genes expression in diverse cancer types.

\section{TIMER database analysis}

TIMER database (https://cistrome.shinyapps.io/timer/) is a website for comprehensive analysis of gene expression and tumor-infiltrating immune cells level of different cancer (41). We used the TIMER database to analyze the differential hub genes expression in tumor and normal tissues in various cancers to validate the results of Oncomine.

\section{Statistical analysis}

Statistical analysis in this study was conducted by $\mathrm{R}$ version 4.0.3. All statistical tests were performed by using a P.value $<0.05$ as the statistically significant difference. Group comparisons were performed with the $t$-test for continuous variables and $\chi^{2}$-test for categorical variables. 


\section{Declarations}

\section{Ethics approval and consent to participated}

Not applicable

\section{Consent for publication}

Not applicable

\section{Data Availability Statement}

The datasets analyzed during the current study are available from the corresponding author Yu Zhang (zhangwkp@163.com) on reasonable request. The data that support the findings of this study are available at the TCGA data portal (https://tcga- data.nci.nih.gov/tcga/), and the comprehensive Gene Expression Omnibus (GEO; https://www.ncbi.nlm.nih.gov/geo/).

\section{Funding}

This work was supported by National Key R\&D Program of China (2017YFC0909900) and National Key R\&D Program of Hubei Province (No.2020BCA060).

\section{Author contributions}

Fang Cheng, Zhendi Wang and Yu Zhang conceived and designed the study. Fang Cheng, Qiang Li, and Jinglin Wang performed the analysis procedures. Zhendi Wang, Fang Zeng, and Yumei Wang analyzed the results. Fang Cheng and Fang Zeng assisted in writing the manuscript. All authors reviewed the manuscript. All authors read and approved the final manuscript.

\section{Acknowledgements}

Not applicable

\section{Conflict of Interest}

The authors have no relevant affiliations or financial involvement with any organization or entity with a financial interest in or financial conflict with the subject matter or materials discussed in the manuscript.

\section{References}

1. Owens B. Kidney cancer. Nature. 2016;537(7620):S97.

2. Cohen HT, McGovern FJ. Renal-cell carcinoma. N Engl J Med. 2005;353(23):2477-90.

3. Srigley JR, Delahunt B, Eble JN, Egevad L, Epstein JI, Grignon D, et al. The International Society of Urological Pathology (ISUP) Vancouver Classification of Renal Neoplasia. Am J Surg Pathol. 
2013;37(10):1469-89.

4. Linehan WM, Ricketts CJ. The Cancer Genome Atlas of renal cell carcinoma: findings and clinical implications. Nat Rev Urol. 2019;16(9):539-52.

5. Escudier B, Porta C, Schmidinger M, Rioux-Leclercq N, Bex A, Khoo V, et al. Renal cell carcinoma: ESMO Clinical Practice Guidelines for diagnosis, treatment and follow-upt. Ann Oncol. 2019;30(5):706-20.

6. Gómez-López G, Valencia A. Bioinformatics and cancer research: building bridges for translational research. Clin Transl Oncol. 2008;10(2):85-95.

7. Yang JF, Shi SN, Xu WH, Qiu YH, Zheng JZ, Yu K, et al. Screening, identification and validation of CCND1 and PECAM1/CD31 for predicting prognosis in renal cell carcinoma patients. Aging (Albany NY). 2019;11(24):12057-79.

8. Lin J, Yu M, Xu X, Wang Y, Xing H, An J, et al. Identification of biomarkers related to CD8(+) T cell infiltration with gene co-expression network in clear cell renal cell carcinoma. Aging (Albany NY). 2020;12(4):3694-712.

9. Mennerich D, Kubaichuk K, Kietzmann T. DUBs, Hypoxia, and Cancer. Trends Cancer. 2019;5(10):632-53.

10. Riera-Domingo C, Audigé A, Granja S, Cheng WC, Ho PC, Baltazar F, et al. Immunity, Hypoxia, and Metabolism-the Ménage à Trois of Cancer: Implications for Immunotherapy. Physiol Rev. 2020;100(1):1-102.

11. Macklin PS, McAuliffe J, Pugh CW, Yamamoto A. Hypoxia and HIF pathway in cancer and the placenta. Placenta. 2017;56:8-13.

12. Semenza GL. Targeting HIF-1 for cancer therapy. Nat Rev Cancer. 2003;3(10):721-32.

13. Vander Heiden MG, DeBerardinis RJ. Understanding the Intersections between Metabolism and Cancer Biology. Cell. 2017;168(4):657-69.

14. Li W, Middha M, Bicak M, Sjoberg DD, Vertosick E, Dahlin A, et al. Genome-wide Scan Identifies Role for AOX1 in Prostate Cancer Survival. Eur Urol. 2018;74(6):710-9.

15. Vantaku V, Putluri V, Bader DA, Maity S, Ma J, Arnold JM, et al. Epigenetic loss of AOX1 expression via EZH2 leads to metabolic deregulations and promotes bladder cancer progression. Oncogene. 2020;39(40):6265-85.

16. Zhang W, Chai W, Zhu Z, Li X. Aldehyde oxidase 1 promoted the occurrence and development of colorectal cancer by up-regulation of expression of CD133. Int Immunopharmacol. 2020;85:106618.

17. Xiong L, Feng Y, Hu W, Tan J, Li S, Wang H. Expression of AOX1 Predicts Prognosis of Clear Cell Renal Cell Carcinoma. Front Genet. 2021;12:683173.

18. Besse A, Wu P, Bruni F, Donti T, Graham BH, Craigen WJ, et al. The GABA transaminase, ABAT, is essential for mitochondrial nucleoside metabolism. Cell Metab. 2015;21(3):417-27.

19. Shin H, Cha HJ, Lee MJ, Na K, Park D, Kim CY, et al. Identification of ALDH6A1 as a Potential Molecular Signature in Hepatocellular Carcinoma via Quantitative Profiling of the Mitochondrial 
Proteome. J Proteome Res. 2020;19(4):1684-95.

20. Zou Y, Lu Q, Yao Q, Dong D, Chen B. Identification of novel prognostic biomarkers in renal cell carcinoma. Aging (Albany NY). 2020;12(24):25304-18.

21. Guo X, Sun Z, Jiang S, Jin X, Wang H. Identification and validation of a two-gene metabolic signature for survival prediction in patients with kidney renal clear cell carcinoma. Aging (Albany NY). $2021 ; 13(6): 8276-89$.

22. Lu J, Chen Z, Zhao H, Dong H, Zhu L, Zhang Y, et al. ABAT and ALDH6A1, regulated by transcription factor HNF4A, suppress tumorigenic capability in clear cell renal cell carcinoma. J Transl Med. 2020;18(1):101.

23. Chen L, Vasoya RP, Toke NH, Parthasarathy A, Luo S, Chiles E, et al. HNF4 Regulates Fatty Acid Oxidation and Is Required for Renewal of Intestinal Stem Cells in Mice. Gastroenterology. 2020;158(4):985-99.e9.

24. Shen C, Song YH, Xie Y, Wang X, Wang Y, Wang C, et al. Downregulation of HADH promotes gastric cancer progression via Akt signaling pathway. Oncotarget. 2017;8(44):76279-89.

25. Wongkittichote P, Ah Mew N, Chapman KA. Propionyl-CoA carboxylase - A review. Mol Genet Metab. 2017;122(4):145-52.

26. Cheng P, Wang Z, Hu G, Huang Q, Han M, Huang J. A prognostic 4-gene expression signature for patients with HER2-negative breast cancer receiving taxane and anthracycline-based chemotherapy. Oncotarget. 2017;8(61):103327-39.

27. Hoesel B, Schmid JA. The complexity of NF-KB signaling in inflammation and cancer. Mol Cancer. 2013;12:86.

28. DiDonato JA, Mercurio F, Karin M. NF-kB and the link between inflammation and cancer. Immunol Rev. 2012;246(1):379-400.

29. Li F, Zhang J, Arfuso F, Chinnathambi A, Zayed ME, Alharbi SA, et al. NF-KB in cancer therapy. Arch Toxicol. 2015;89(5):711-31.

30. Eke I, Cordes N. Focal adhesion signaling and therapy resistance in cancer. Semin Cancer Biol. 2015;31:65-75.

31. Paluch EK, Aspalter IM, Sixt M. Focal Adhesion-Independent Cell Migration. Annu Rev Cell Dev Biol. 2016;32:469-90.

32. O'Shea JJ, Schwartz DM, Villarino AV, Gadina M, Mclnnes IB, Laurence A. The JAK-STAT pathway: impact on human disease and therapeutic intervention. Annu Rev Med. 2015;66:311-28.

33. Owen KL, Brockwell NK, Parker BS. JAK-STAT Signaling: A Double-Edged Sword of Immune Regulation and Cancer Progression. Cancers (Basel). 2019;11(12).

34. Barrett T, Wilhite SE, Ledoux P, Evangelista C, Kim IF, Tomashevsky M, et al. NCBI GEO: archive for functional genomics data sets-update. Nucleic Acids Res. 2013;41(Database issue):D991-5.

35. Huang da W, Sherman BT, Lempicki RA. Bioinformatics enrichment tools: paths toward the comprehensive functional analysis of large gene lists. Nucleic Acids Res. 2009;37(1):1-13. 
36. Xie C, Mao X, Huang J, Ding Y, Wu J, Dong S, et al. KOBAS 2.0: a web server for annotation and identification of enriched pathways and diseases. Nucleic Acids Res. 2011;39(Web Server issue):W316-22.

37. Szklarczyk D, Franceschini A, Wyder S, Forslund K, Heller D, Huerta-Cepas J, et al. STRING v10: protein-protein interaction networks, integrated over the tree of life. Nucleic Acids Res. 2015;43(Database issue):D447-52.

38. Assenov Y, Ramírez F, Schelhorn SE, Lengauer T, Albrecht M. Computing topological parameters of biological networks. Bioinformatics. 2008;24(2):282-4.

39. Powers RK, Goodspeed A, Pielke-Lombardo H, Tan AC, Costello JC. GSEA-InContext: identifying novel and common patterns in expression experiments. Bioinformatics. 2018;34(13):i555-i64.

40. Rhodes DR, Yu J, Shanker K, Deshpande N, Varambally R, Ghosh D, et al. ONCOMINE: a cancer microarray database and integrated data-mining platform. Neoplasia. 2004;6(1):1-6.

41. Li T, Fan J, Wang B, Traugh N, Chen Q, Liu JS, et al. TIMER: A Web Server for Comprehensive Analysis of Tumor-Infiltrating Immune Cells. Cancer Res. 2017;77(21):e108-e10.

\section{Figures}

A

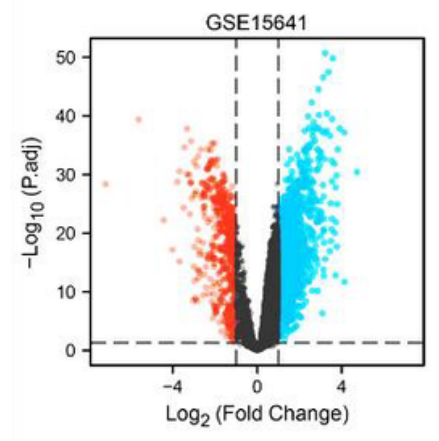

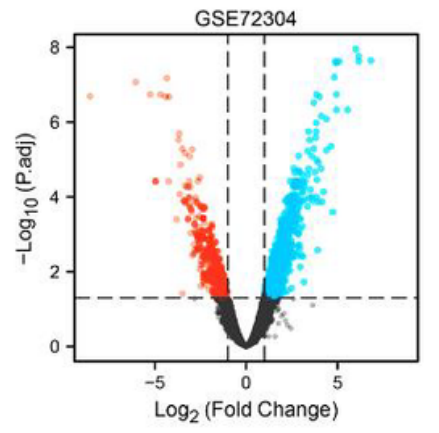
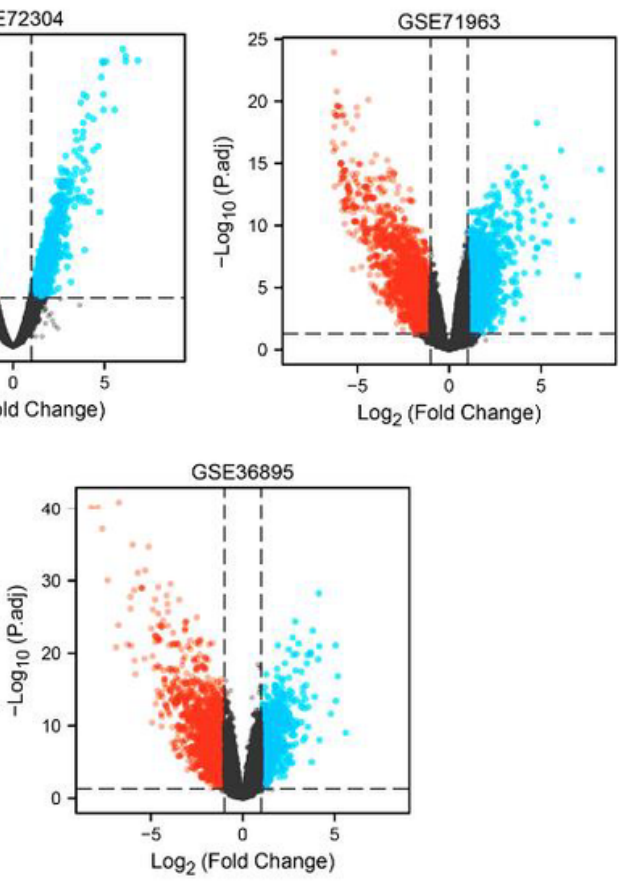

B

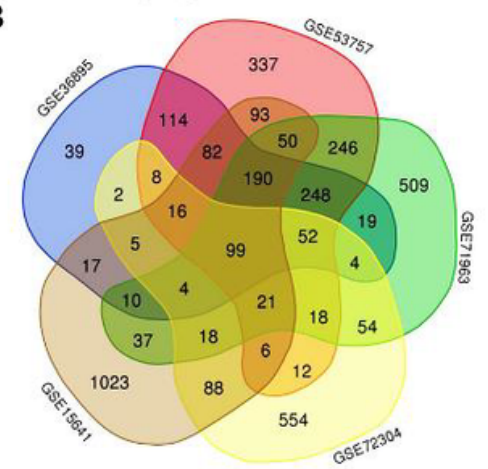

$\mathrm{C}$

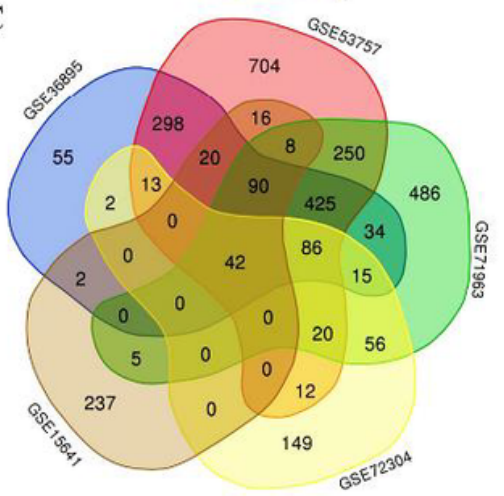

Downregulated DEGs

\section{Figure 1}

Identification of common DEGs in KIRC. A. Volcano plots of gene expression profile data in GSE15641, GSE72304, GSE71963, GSE53757, and GSE36895, respectively. B. Venn plots of upregulated common 
DEGs. C. Venn plots of downregulated common DEGs.

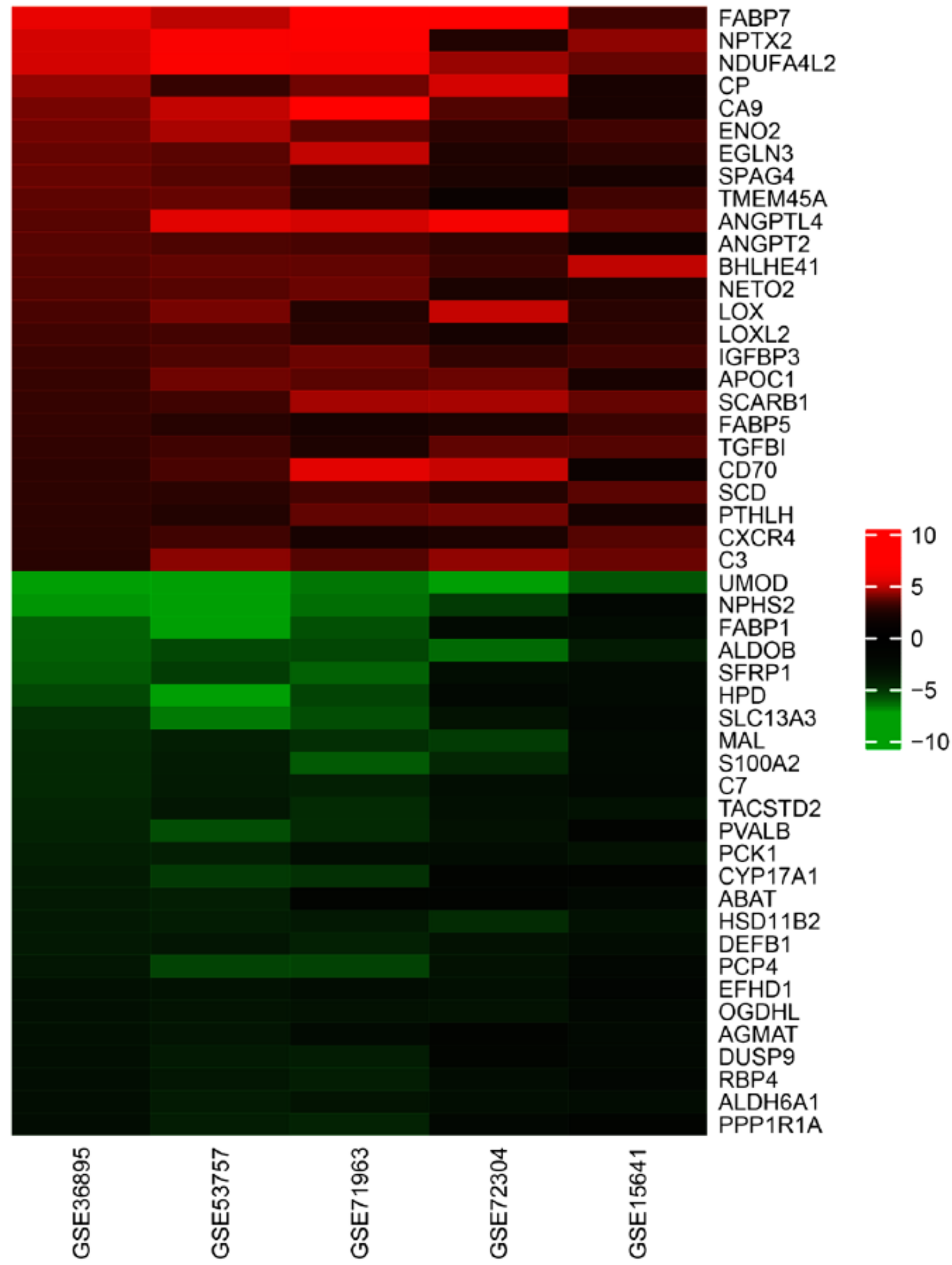

Figure 2

The heat map of top 25 upregulated and downregulated DEGs in the integrated microarray analysis. Each column represents one GEO ID and each row represents one gene name. The value in each rectangle expresses the value of the log2FC value. Log2FC $>0$ expresses upregulated and log2FC $<0$ expresses downregulated. 
A

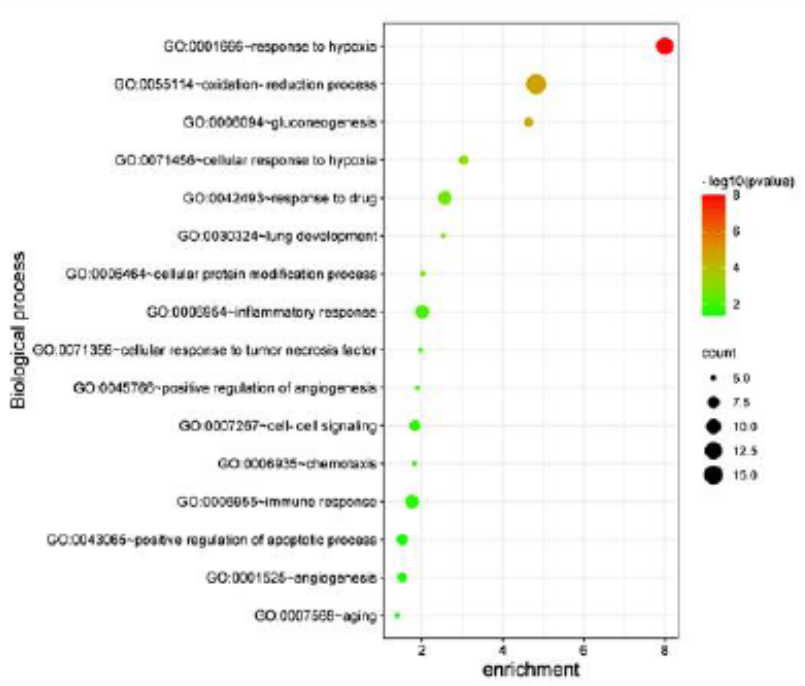

$\mathrm{C}$

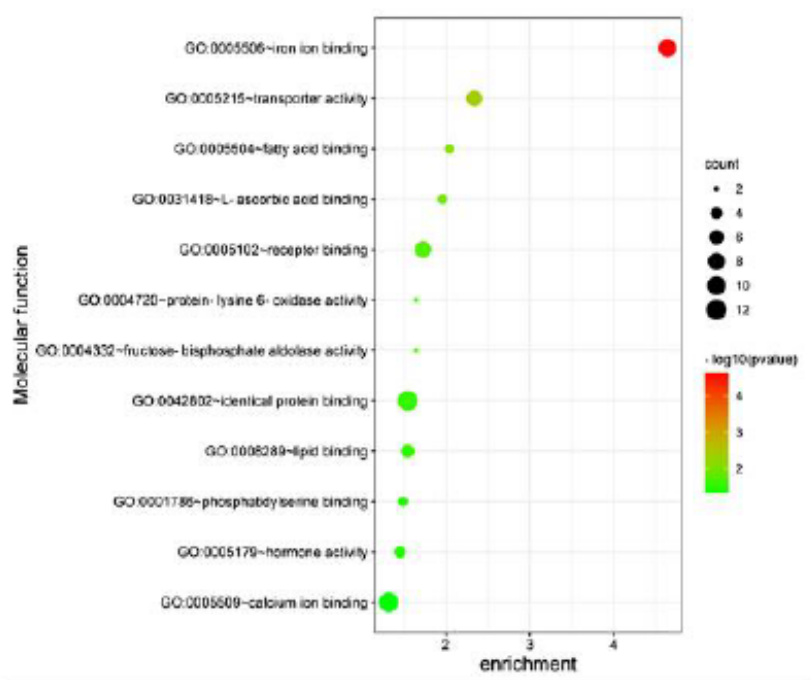

B

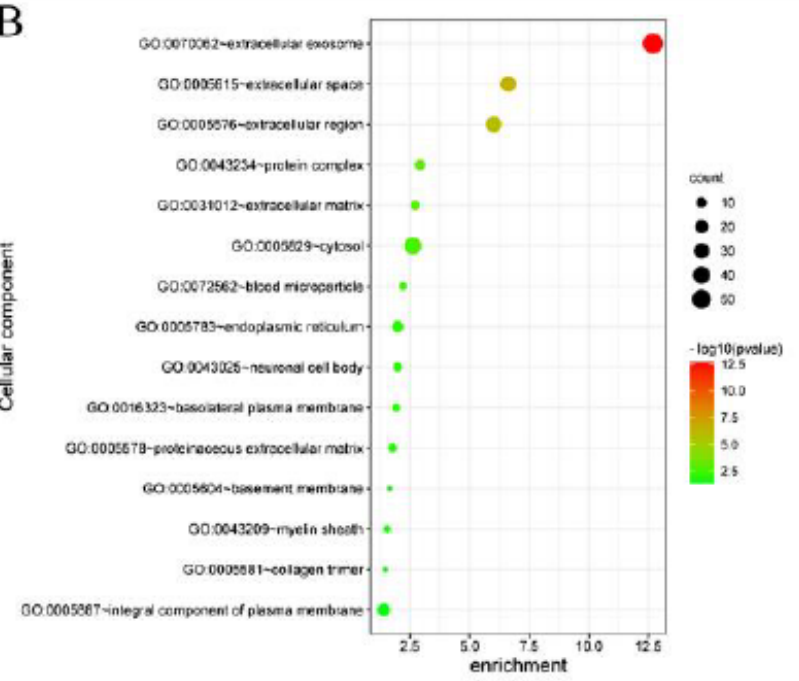

D

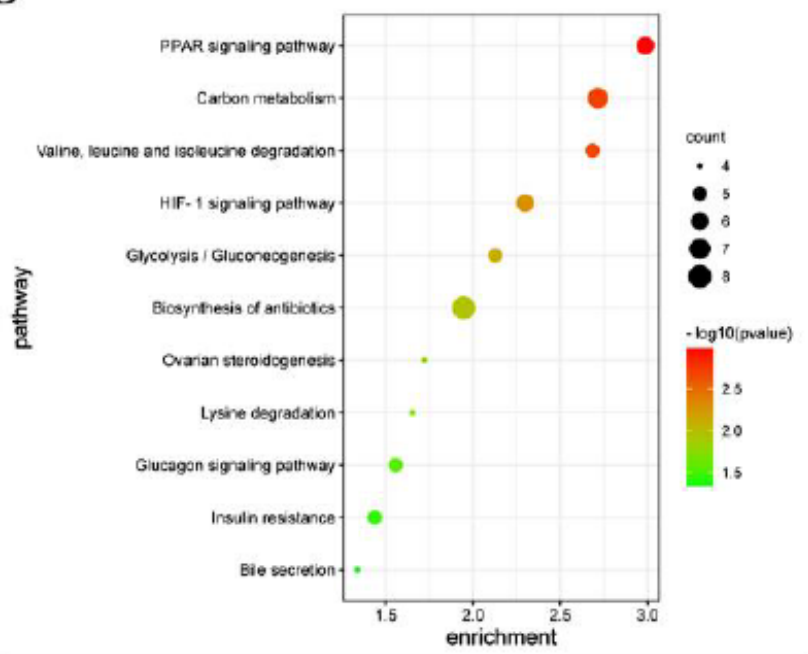

\section{Figure 3}

GO and KEGG analysis of the DEGs. A. Biological process. B. Cell component. C. Molecular function. D. KEGG pathway. The y-axis shows significantly enriched categories of the targets, and the $x$-axis shows the enrichment scores of these terms. Bubble size denotes the number of genes located in the functional area. DEG, differentially expressed genes; GO, Gene Ontology; KEGG, Kyoto Encyclopedia of Gene and Genome 
A

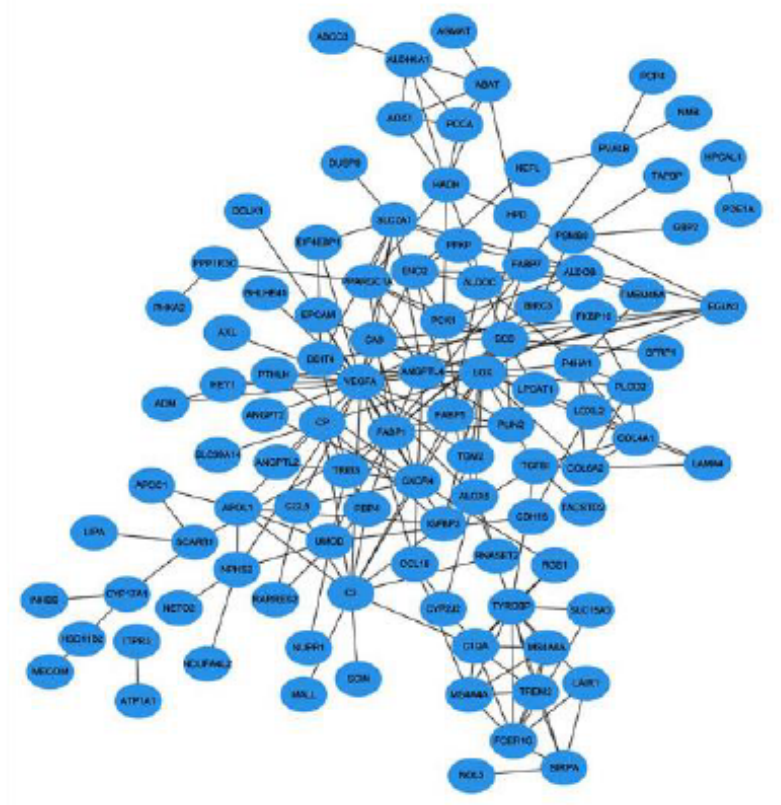

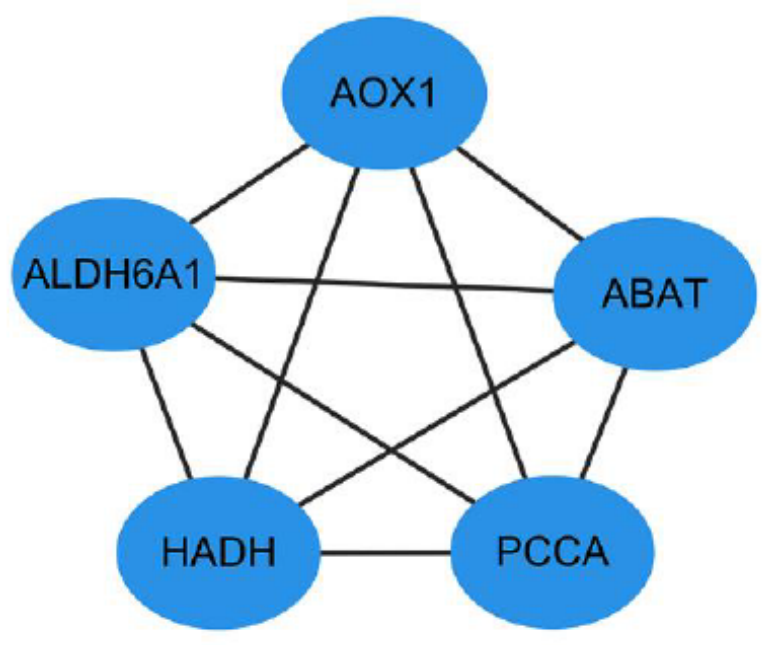

Figure 4

The PPI network of DEGs. A. The PPI network of the DEGs. B. Top 5 hub genes screened with Cytotype MCODE.

A
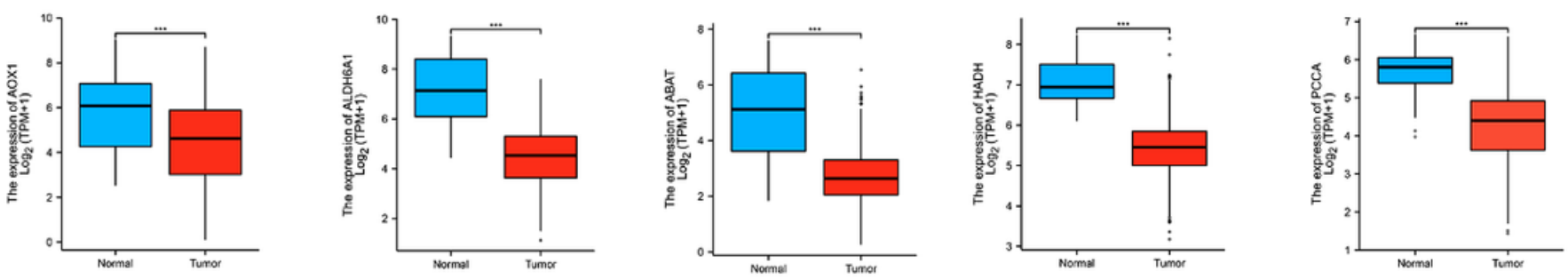

$\mathrm{B}$
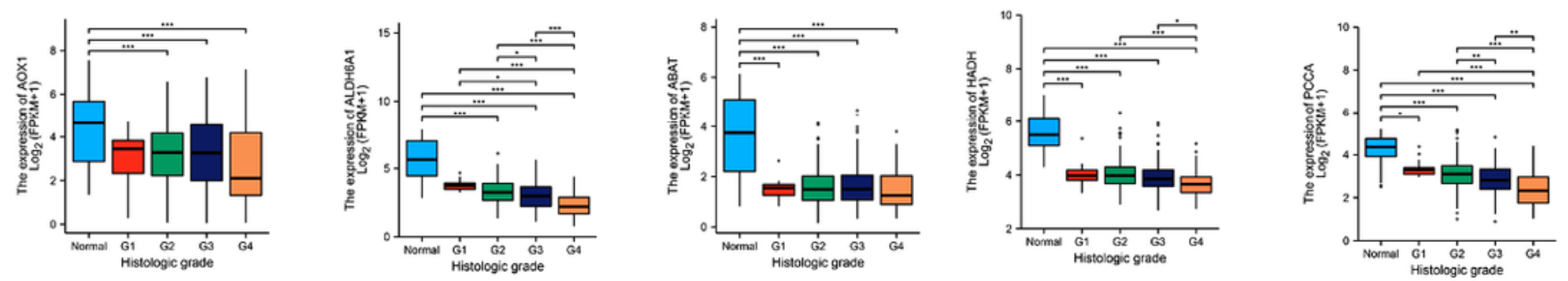

C
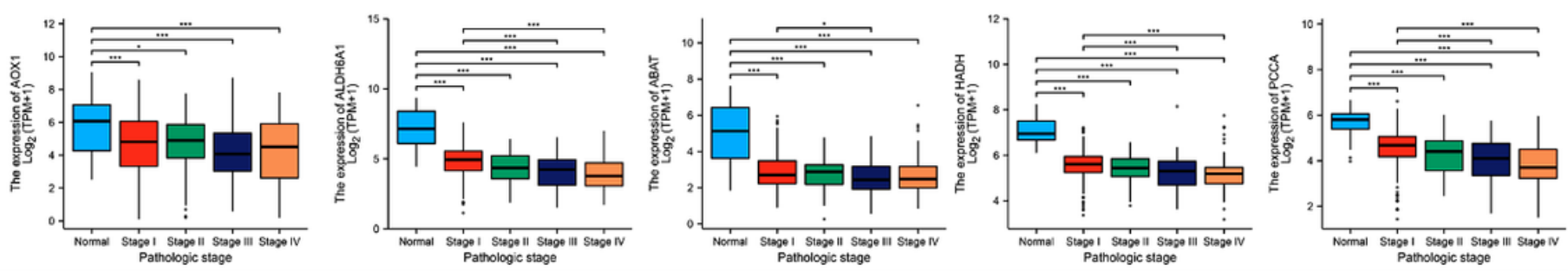

Figure 5 
Differential expression of hub genes in different clinical characteristics. (A) Hub genes expression difference in KIRC samples and normal kidney samples. (B) tumor histology stage. (C) pathological stage.

A
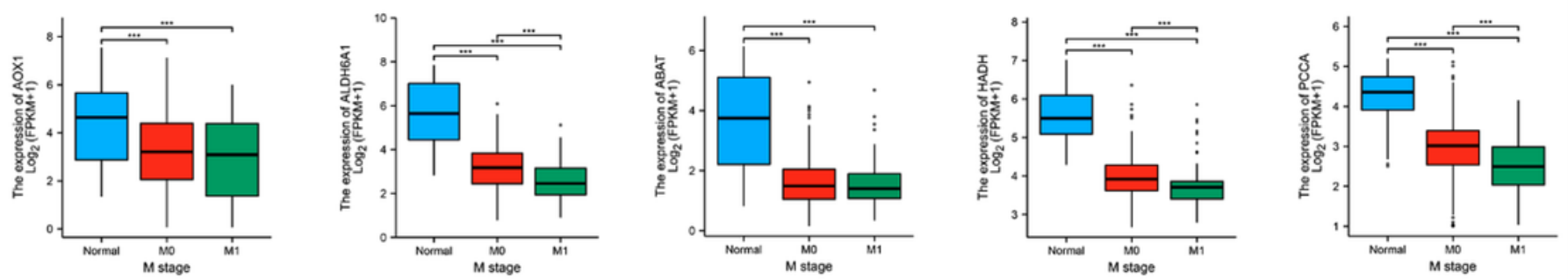

B
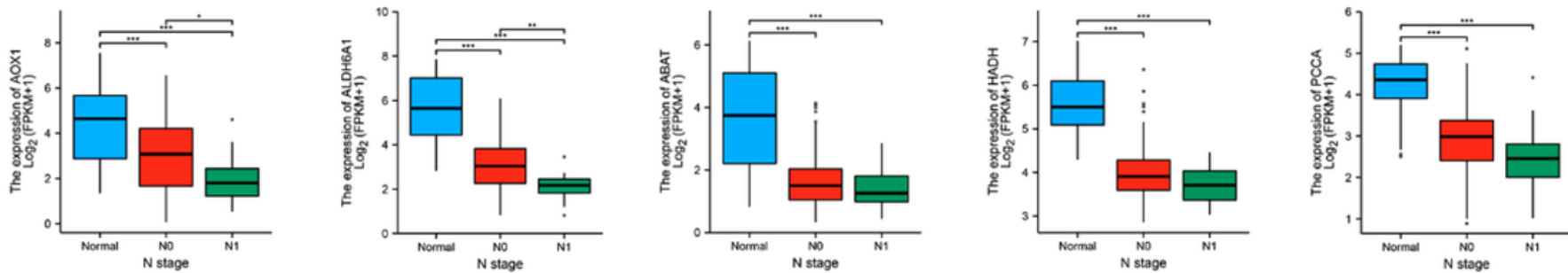

C
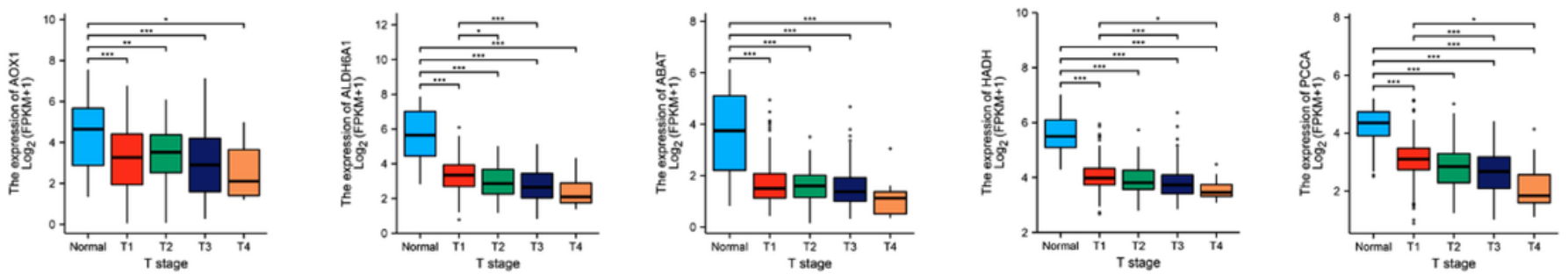

Figure 6

Differential expression of hub genes in different clinical characteristics. (A) Distant metastasis. (B) Lymph node metastasis. (C) Tumor status.

A
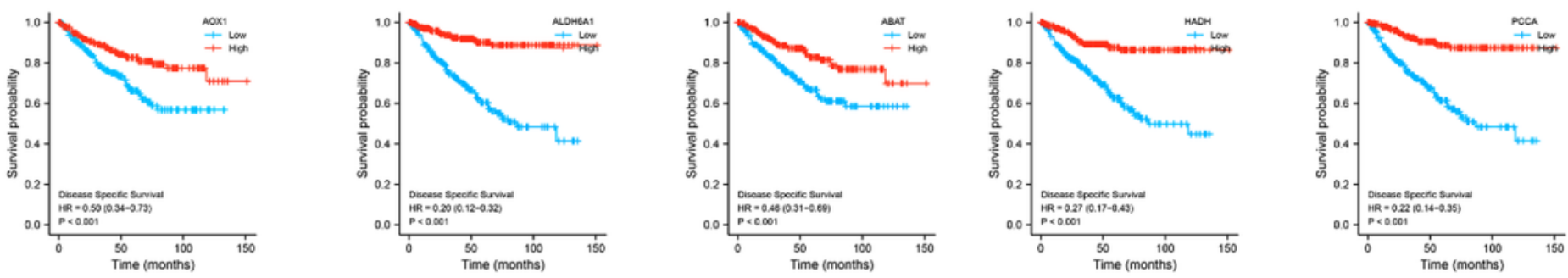

B
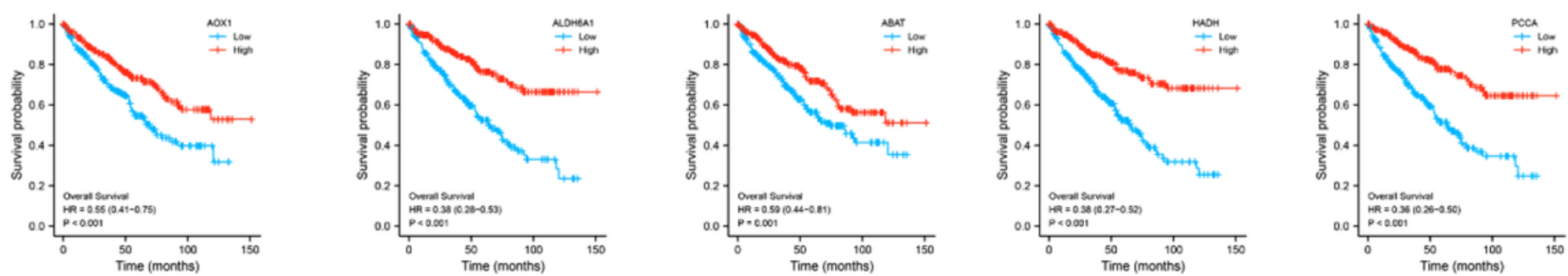
Figure 7

Prognostic significance of hub genes in KIRC. (A) Over survival. (B) Disease specific survival.

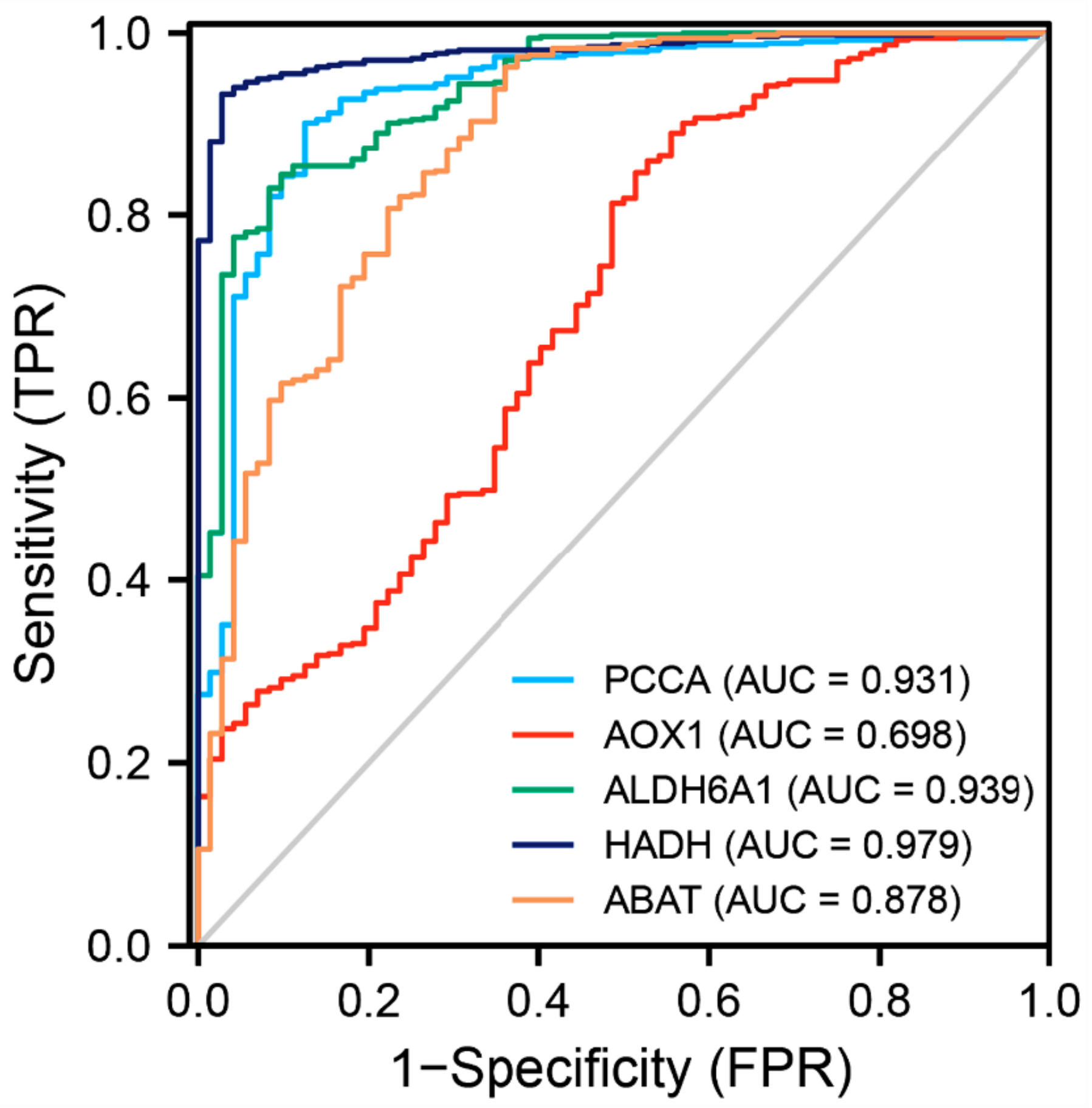

Figure 8

ROC curve of hub gene. 
AOX1

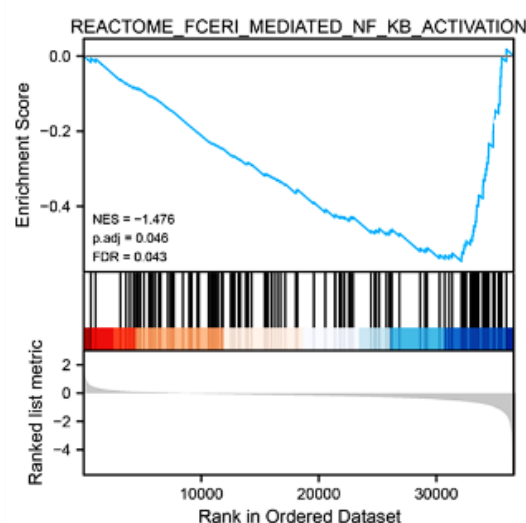

ALDH6A1

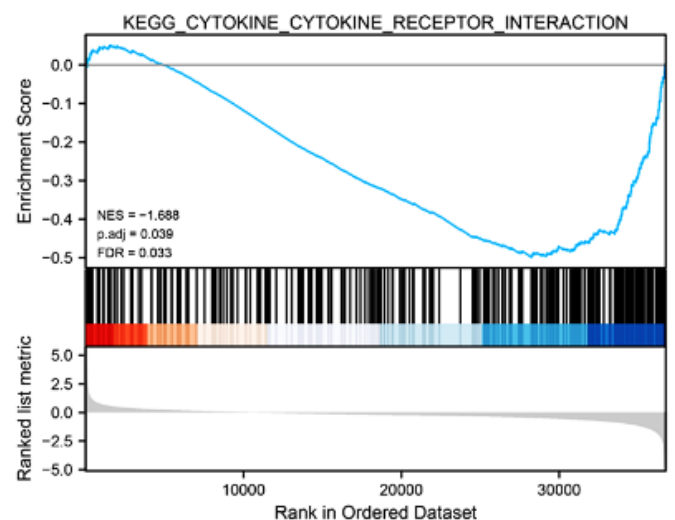

ABAT

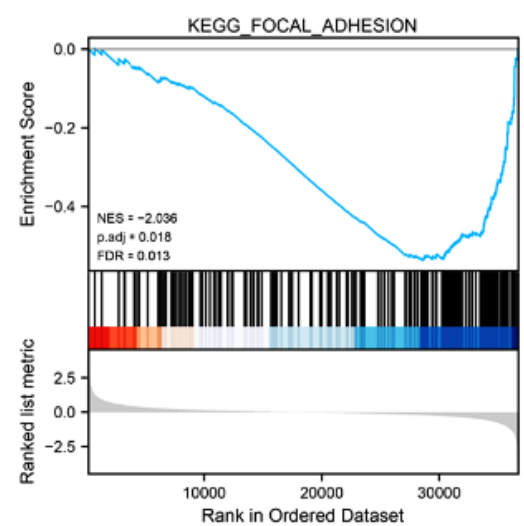

PCCA

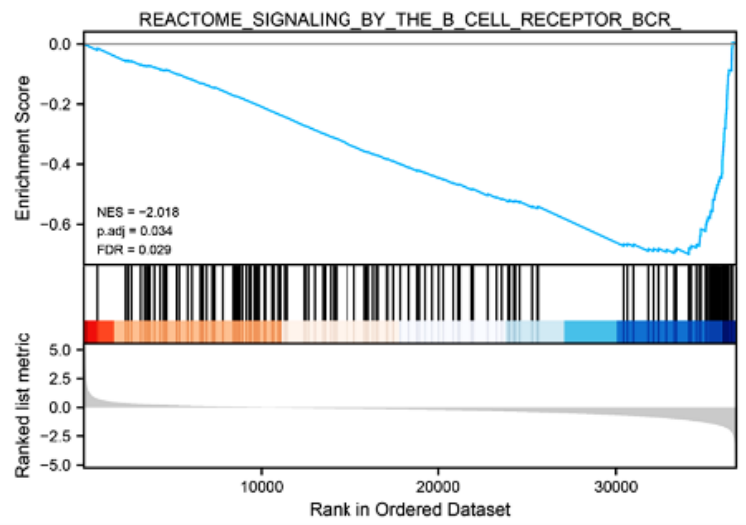

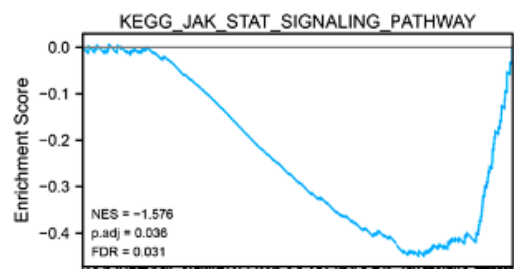

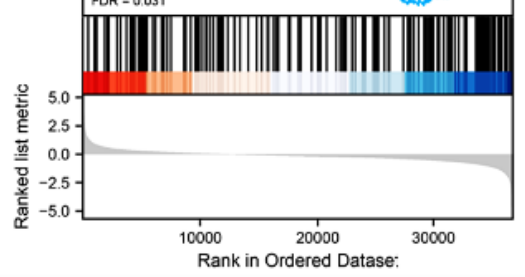

Figure 9

GSEA analysis of hub genes.

A

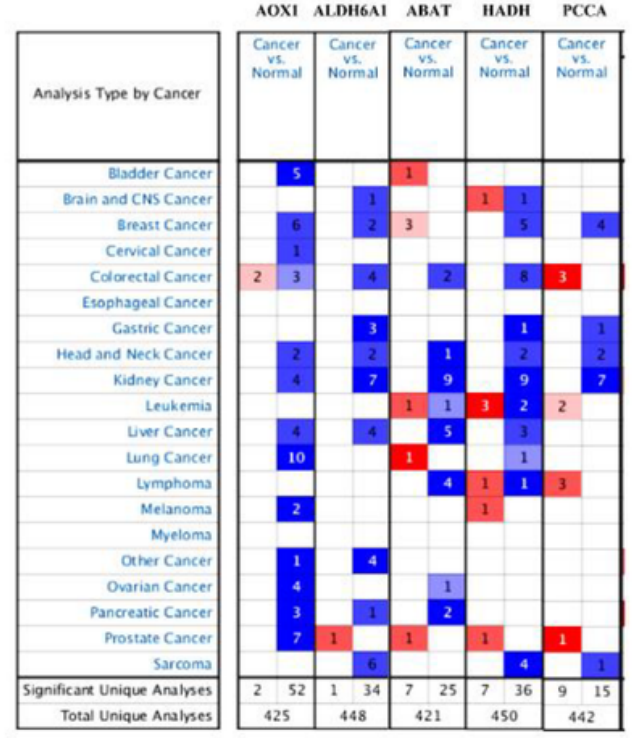

B
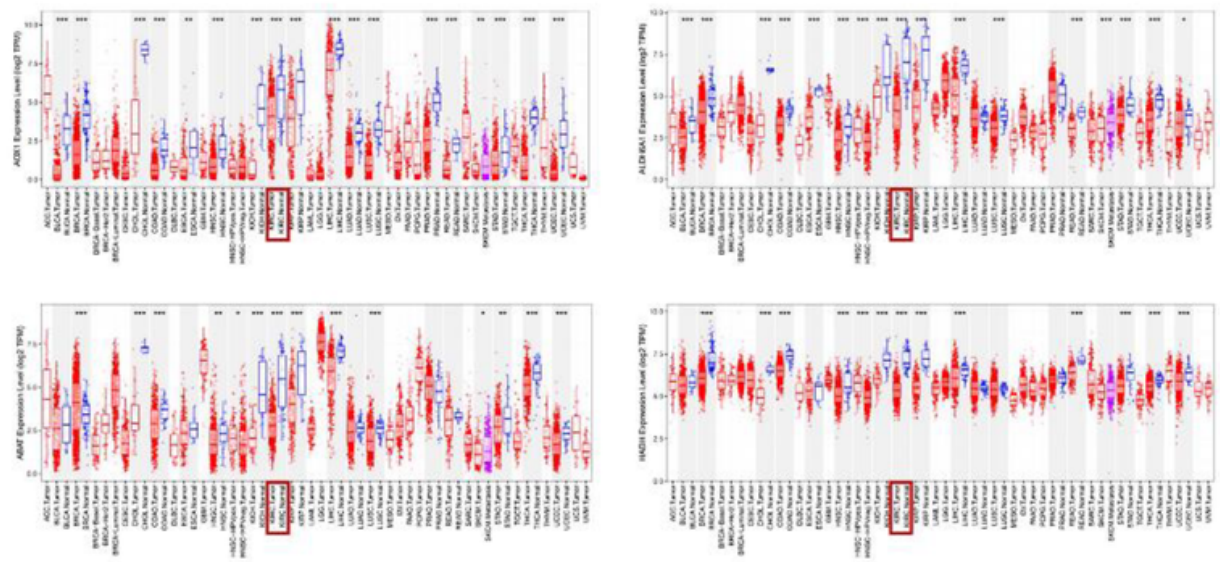

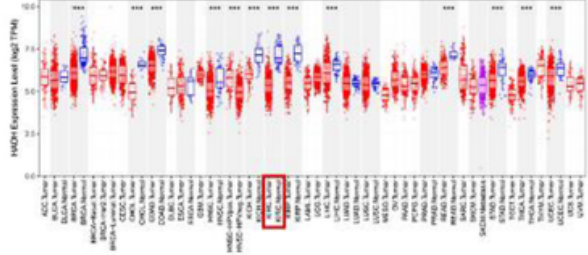


Figure 10

The expression of hub genes in different cancers. (A) Hub genes level in different cancers tissues compared to normal tissues in the Oncomine database. (B) Hub genes expression of different tumor types in the TIMER database. 